\title{
1 Hybrid In Situ Product Recovery technique applied to (A)IBE Fermentation
}

2 B. Pérez-Bibbins ${ }^{(1)}$, H. Gonzalez Peñas ${ }^{(1)^{*}}$, E. Toth $^{(1)}$, V. Coupard ${ }^{(1)}$, N. Lopes-Ferreira 3 (2)

4

5 (1)IFP Energies Nouvelles, Rond-point de l'échangeur de Solaize BP 3, 69360 Solaize, 6 France

7 (2)IFP Energies Nouvelles, 232, Avenue Napoléon Bonaparte, 92852, Rueil-Malmaison, 8 France

9

*Corresponding author: helena.gonzalez-penas@ifpen.fr

10 


\section{Abstract}

12 Fermentative production of alcohols is a promising alternative to petroleum-based fuels

13 industry. However, the development of a competitive biological process implies the

14 achievement of higher production titer and enhanced productivity. In the case of butanol

15 production by solventogenic strains, In Situ Product Recovery (ISPR) techniques help

16 to overcome the inhibition threshold of the microorganism and to reduce the overall cost

17 of downstream process. In this work, gas stripping (GS) was preliminary studied in an

18 abiotic system and high flow rates (vvm - vessel volume minute- $>2 \mathrm{~min}^{-1}$ ) were needed

19 to achieve higher butanol stripping rate than its production rate. Combined liquid

20 extraction (LE) with pulse Gas Stripping (GS) were applied to batch fermentations

21 resulting in a synergic effect giving a higher glucose consumption rate and a lower

22 aqueous butanol concentration. Moreover, in this hybrid system, biphasic bioreactor

23 acted as a "liquid-liquid equilibrium step" and both phases were stripped concomitantly.

25 Keywords: IBE; extractive fermentation; gas stripping; Clostridium beijerinckii. 


\section{Introduction}

Production of fuels and chemicals from sugars has attracted much attention during the last decades, becoming a global priority as atmospheric $\mathrm{CO}_{2}$ levels and oil prices continue to increase. Alcohols obtained by fermentation processes are included among the most promising petroleum substitutes due to their wide range of applications: advanced biofuels, industrial solvents, chemical feedstock, bioplastics production, etc.

The development of an economically competitive biological process implies the achievement of higher production titers of alcohol in the bioreactor. However, microorganisms usually have limited tolerance for certain products and may be inhibited by excessively high concentrations occurring when the threshold concentration is exceeded. Particularly, ABE (acetone, butanol, ethanol) and IBE (isopropanol, butanol, ethanol) fermentations are subject to strong inhibition by products [1], which leads to low final product concentration, high recovery costs and large volumes of wastewater generation. Several options have been reported to produce metabolites from fermentation beyond their inhibition threshold. One possible solution would deal with the improvement of the biocatalyst, in terms of higher selectivity and tolerance towards the main fermentation product. Another alternative focuses on the biocatalyst environment and concerns the decrease of product inhibition by continuous product removal and recovery from the fermentation medium. This can be achieved by an In Situ Product Removal system. The later approach allows the recovery of butanol as fast as it is produced during the fermentation, and thus it would keep the butanol concentration under the inhibition threshold. This allows the generation of more concentrated butanol stream which is more easily recovered $[2,3,4,5,6]$ and results in several benefits impacting the overall process performance: smaller reactor volume, 
51 lower downstream cost, less wastewater generation. The integrated product recovery of 52 butanol (and other alcohols) from aqueous broth can be based on the differences between physical and chemical properties of the compounds to be separated, or on their interaction with an auxiliary agent or material. In any case, the total recovery of butanol is not necessarily achieved in the pre-concentration step and thus further purification may be required. Separation techniques for in situ product recovery that have been explored over the last few decades are adsorption, gas stripping, liquid-liquid extraction, pervaporation, etc.

Among different possibilities, gas stripping (GS) arises as a relatively simple technique that can effectively remove AIBE compounds from the fermentation broth [7]. Gases generated during AIBE fermentation -or external agents like nitrogen - are used in this technique to separate the solvents. These gases are sparged into the bioreactor and volatile compounds are recovered and subsequently condensed. Gas stripping can be used either in situ or in stream configurations, with or without previous removal of solids. Several AIBE configurations (batch, fed batch and continuous mode) have been tested in laboratory scale, and in all cases both the product yield and productivity have improved. The effects of several operating parameter such as the gas recycle rate, the bubble size or the amount of antifoam added to the broth have been studied $[8,9]$ for a gas stripping system coupled or not to fermentation. Ezeji et al., 2005 [10] observed that C. beijerinckii was not affected adversely by GS and productivities and yields were 71 increased to 200 and $118 \%$ respectively, as compared to control in batch fermentation. 72 Xue et al., 2012 [11] applied gas stripping in fed-batch mode to a system combining free and immobilized cells doubling its reference productivity, obtaining highly concentrated condensates and reducing about $90 \%$ of the estimated energy cost for the 
overall process. However, even if GS technique improves in all cases fermentation performances, before its industrial application, several aspects will need to be faced up, such as: excessive foaming during fermentation that leads to operational problems, high cost of stripped alcohols recovery, huge compressors needed.

In situ butanol recovery technique by liquid extraction (LE) coupled to a fermentation system, an insoluble extracting compound is added to the fermentation broth. Both phases are then easily separated. The optimal solvent is a compromise between high alcohol distribution coefficient and no toxicity towards microorganisms [6, 12]. In practice, biocompatible solvents present low butanol extraction capacity, therefore high volumetric ratio of solvents to aqueous phase is needed in order to keep butanol below the inhibition threshold concentration, which increases the operational volume necessary in the bioreactor.

Combination of both in situ recovery techniques (GS and LE) and their applications for batch and fed-batch ABE fermentations were first proposed by Lu and Li, 2016 [13]. In their work, the authors applied nitrogen stripping directly through the organic phase (oleyl alcohol) in a biphasic reactor. They obtained an ABE productivity of $40 \%$ higher at $\mathrm{vvm}=1.6 \mathrm{~min}^{-1}$ in batch mode and increased up to $95-105 \%$ in fed batch mode [14] applying 1.6 and $3.3 \mathrm{~min}^{-1}$ of gas flow rate, respectively. Moreover, glucose consumption was boosted consequently.

In this work, a hybrid system combining gas stripping and liquid extraction was applied to batch IBE fermentations. Vegetable oil based mixture solvent was used in a biphasic reactor and the integrated technique (GS-LLE) performance was compared to control assays (individual ISPR technique). Previously, gas stripping was studied in an abiotic set up (representative synthetic fermentation medium, no cells) in order to evaluate a 
99 first order striping rate model and to establish the optimal operating window for this 100 system.

1. Material and Methods

102

\subsection{Experimental set-up for abiotic IBE gas stripping}

103

A schematic of the experimental apparatus for gas stripping used in this study is shown in Fig. 1. End fermentation representative solutions of 1-butanol-isopropanol-ethanol (11/5/0.5 g/L) (PubChem CID: 263, 3776, 702, respectively) were prepared in demineralized water and placed in $1 \mathrm{~L}$ bioreactor $(500 \mathrm{~mL}$ of working volume) with a Rushton impeller and a sparger placed on the bottom of the vessel below the impeller. Temperature inside the bioreactor, agitation rate and gas flow rate were controlled variables in the system. For collecting condensates, two cold traps were arranged and cooled at 4 and $-15^{\circ} \mathrm{C}$, respectively. Sampling was done in the reactor and also in both cold traps, so the performance of total alcohol stripping process could be estimated. Nitrogen (PubChem CID: 947) was sparged through the aqueous solution at a fixed flow rate before it was led to the condenser flasks and then through a flask with water containing ice before it was released to the atmosphere. Temperature set point in the bioreactor was fixed at $37{ }^{\circ} \mathrm{C}$ while agitation rate was set at $300 \mathrm{rpm}$. Gas stripping rate was evaluated at several vvm $\left(1 \mathrm{vvm}=1 \mathrm{~L}\right.$ of $\mathrm{N}_{2}$ per $1 \mathrm{~L}$ of liquid volume per minute):

$1170.5,2$ and $3 \mathrm{~min}^{-1}$. Each experiment was carried out twice.

\subsection{Mathematical development}

The system was described by means of a simple macroscopic model in order to quantify the stripping process. At gas-liquid interface, the steady state flux balance can be expressed as follows according to Whitman double film theory: 


$$
\phi_{\text {fluxGL }}=\mathrm{k}_{\mathrm{L}}\left(\mathrm{C}_{\mathrm{i}, 1}-\mathrm{C}_{\mathrm{i}, 1}^{*}\right)=\mathrm{k}\left(\mathrm{C}_{\mathrm{i}, \mathrm{g}}^{*}-\mathrm{C}_{\mathrm{i}, \mathrm{g}}\right)=\mathrm{K}_{\mathrm{LG}}\left(\mathrm{HC}_{\mathrm{i}, 1}-\mathrm{C}_{\mathrm{i}, \mathrm{g}}^{*}\right) \quad\left(\mathrm{mol} / \mathrm{m}^{2} \mathrm{~s}\right) \quad \text { Eq. A.1 }
$$

Where $k_{L}, k_{G}$ are respectively the individual mass transfer coefficient based on liquid and gas phases $(\mathrm{m} / \mathrm{s}), K L_{G}$ is the overall mass transfer coefficient based in gas phase $(\mathrm{m} / \mathrm{s}), C_{i, l}, C_{i, g}$ the bulk liquid and gas composition for compound $\mathrm{i}\left(\mathrm{mol} / \mathrm{m}^{3}\right)$,

126 respectively. $C_{i, l}^{*}, C_{i, g}^{*}$ are the corresponding equilibrium composition with bulk 127 composition in each phase $\left(\mathrm{mol} / \mathrm{m}^{3}\right)$. Moreover, $\mathrm{H}$ denotes the Henry coefficient 128 (dimensionless). Therefore, the gas phase balance is expressed as follows:

$$
\mathrm{V}_{\mathrm{g}} \frac{\mathrm{d}\left(\mathrm{C}_{\mathrm{i}, \mathrm{g}}\right)}{\mathrm{dt}}=-\mathrm{Q}_{\mathrm{g}}\left(\mathrm{C}_{\mathrm{i}, \mathrm{g}}\right)+\mathrm{V}_{\mathrm{T}} \mathrm{K}_{\mathrm{LG}} a\left(\mathrm{HC}_{\mathrm{i}, \mathrm{l}}-\mathrm{C}_{\mathrm{i}, \mathrm{g}}^{*}\right) \quad(\mathrm{mol} / \mathrm{s}) \quad \text { Eq. A. } 2
$$

130 Where $V_{g}$ is the gas phase volume in the bioreactor $\left(\mathrm{m}^{3}\right), V_{T}$ the total volume $\left(\mathrm{m}^{3}\right), Q_{g}$ 131 denotes the flow gas rate through the bioreactor $\left(\mathrm{m}^{3} / \mathrm{h}\right)$. Gas composition is assumed to 132 be solute free at the bioreactor inlet. Eq.A.2 may be simplified with a quasi-steady state 133 approximation in the gas phase as following:

$\mathrm{C}_{\mathrm{i}, \mathrm{g}}=\frac{\mathrm{V}_{\mathrm{T}} \mathrm{K}_{\mathrm{LG}} a H}{\mathrm{Q}_{\mathrm{g}}+\mathrm{V}_{\mathrm{T}} \mathrm{K}_{\mathrm{LG}} a} \mathrm{C}_{\mathrm{i}, \mathrm{I}}$ $\left(\mathrm{mol} / \mathrm{m}^{3}\right)$ ...Eq. A.3

In liquid phase, mass balance for solute $i$ being stripped can be expressed as:

$$
\mathrm{V}_{1} \frac{\mathrm{d}\left(\mathrm{C}_{\mathrm{i}, 1}\right)}{\mathrm{dt}}=\left(-\mathrm{r}_{\mathrm{i}}\right) \mathrm{V}_{1}+\mathrm{Q}\left(\mathrm{C}_{\mathrm{i}, 1,0}-\mathrm{C}_{\mathrm{i}, \mathrm{l}}\right)-\mathrm{V}_{\mathrm{T}} \mathrm{K}_{\mathrm{LG}} a\left(\mathrm{HC}_{\mathrm{i}, 1}-\mathrm{C}_{\mathrm{i}, \mathrm{g}}\right)\left(\mathrm{mol} / \mathrm{m}^{3}\right) \quad \text { Eq.A.4 }
$$

137 Where $V_{l}$ is the liquid phase volume in the bioreactor $\left(\mathrm{m}^{3}\right), V_{T}$ the total volume 138 (therefore $\left.V_{T}=V_{l}+V_{g}\right)\left(\mathrm{m}^{3}\right), Q$ denotes the liquid flow rate through the bioreactor $\left(\mathrm{m}^{3} / \mathrm{h}\right)$ 139 and $r_{i}$ the reaction rate for compound $\mathrm{i}$ inside the bioreactor (mol/Lh). The first two 140 terms are assumed to be equal to zero (closed system for liquid phase, and no reaction 141 inside since abiotic system was employed). Considering these assumptions and 
142 combining previous equations, the simplified expression for the variation of aqueous 143 phase composition during gas stripping for compound I was obtained:

$144 \frac{\mathrm{d}\left(\mathrm{C}_{\mathrm{i}, 1}\right)}{\mathrm{dt}}=-\frac{\mathrm{K}_{\mathrm{LG}} a Q_{g} H}{\frac{\mathrm{V}_{\mathrm{L}}}{\mathrm{V}_{\mathrm{T}}} Q_{g}+\mathrm{V}_{\mathrm{L}} \mathrm{K}_{\mathrm{LG}} a} \mathrm{C}_{\mathrm{i}, 1}=-\beta \mathrm{C}_{\mathrm{i}, 1} \quad\left(\mathrm{~mol} / \mathrm{m}^{3} \mathrm{~s}\right) \quad$ Eq. A.5

$145 \beta$ denotes the stripping factor which finally encloses thermodynamics $(H)$ and transfer $146\left(K_{L G} a\right)$ effects. If we assume that the system does not have any mass transfer limitations, 147 previous equation may be simplified to:

$148 \frac{\mathrm{d}\left(\mathrm{C}_{\mathrm{i}, 1}\right)}{\mathrm{dt}}=-\frac{Q_{g} H}{\mathrm{~V}_{\mathrm{L}}} \mathrm{C}_{\mathrm{i}, 1}=-\beta \mathrm{C}_{\mathrm{i}, 1} \quad\left(\mathrm{~mol} / \mathrm{m}^{3} \mathrm{~s}\right) \quad$ Eq. A.6

149 According to Eq. A.6, in a thermodynamic controlled system stripping factor can only 150 be improved by applying higher vessels volume per minute to the system.

151 In parallel, dynamic simulation of a successive liquid-vapor flash operations occurring 152 inside the bioreactor has been developed with SIMULIS Thermodynamics software. 153 The aim of this model is the prediction of the system thermodynamics, in other words, 154 the maximal attainable stripping rate at operating conditions, in case of no gas-liquid 155 transfer limitations exist. This model also includes liquid-liquid-vapor flash calculus 156 applied to outlet gas flow, in order to simulate cold trap units and to predict condensates 157 composition. 


\subsection{Microorganism and culture media}

160

C. beijerinckii DSMZ 6423 spores were stored in $150 \mu \mathrm{L}$ saline suspension cryotubes at

$-60{ }^{\circ} \mathrm{C}$. All experiments started with a heat-shock of the spores for $1 \mathrm{~min}$ at $100{ }^{\circ} \mathrm{C}$ to induce germination and subsequently they were used to inoculate $10 \mathrm{ml}$ of 163 potato/glucose preculture medium previously sterilized $\left(121{ }^{\circ} \mathrm{C}, 20 \mathrm{~min}\right)$. The 164 preculture media was incubated anaerobically at $36{ }^{\circ} \mathrm{C}, 24 \mathrm{~h}$. The abiotic culture medium was regenerated at $100{ }^{\circ} \mathrm{C}$ for $10 \mathrm{~min}$ previously and placed inside an Anaerocult jar (Oxoid) for $72 \mathrm{~h}$ to guarantee anoxic conditions at the beginning of the fermentation. The potato/glucose preculture media contained the following composition: $250 \mathrm{~g} / \mathrm{L}$ boiled potatoes; $2 \mathrm{~g} / \mathrm{L}\left(\mathrm{NH}_{4}\right)_{2} \mathrm{SO}_{4}$ (PubChem CID:6097028); 2 g/L CaCO 3 (PubChem CID: 10112), 10 g/L glucose (PubChem CID: 53782692) . The pre-culture medium was transferred to $100 \mathrm{ml}$ of culture medium disposed in $250 \mathrm{~mL}$ sealed biphasic bioreactor using Schott bottle, previously purged with nitrogen (PubChem CID: 947) during $20 \mathrm{~min}$. The culture medium composition for IBE fermentations was: 6 mg/L FeSO $47 \mathrm{H}_{2} \mathrm{O}$ (PubChem CID: 62662); $1 \mathrm{~g} / \mathrm{L} \mathrm{MgSO} 47 \mathrm{H}_{2} \mathrm{O}$

174 (PubChem CID: 24083); 1 g/L KH $\mathrm{KH}_{2} \mathrm{PO}_{4}$ (PubChem CID: 516951); $0.6 \mathrm{~g} / \mathrm{L} \mathrm{K} \mathrm{K}_{2} \mathrm{HPO}_{4}$ 175 (PubChem CID: 24450); 2.4 g/L $\mathrm{CH}_{3} \mathrm{COONH}_{4}$ (PubChem CID: 176); 0.1 g/L paminobenzoic (PubChem CID: 978); 2.5 g/L yeast extract (PubChem CID: 24973165) and $60 \mathrm{~g} / \mathrm{L}$ glucose (PubChem CID: 53782692). Biphasic bioreactors were incubated at $36{ }^{\circ} \mathrm{C}$ and low orbital agitation $(50 \mathrm{rpm})$. 
Batch IBE fermentations were performed to study and compare the effect of pulsed gas stripping, liquid liquid extraction and integrated gas stripping-liquid liquid extraction system. All fermentations were carried out in $500 \mathrm{~mL}$ schott bottles, filled with $200 \mathrm{~mL}$ of culture media and initial glucose concentration of $90 \mathrm{~g} / \mathrm{L}$. The culture medium was inoculated with $20 \mathrm{~mL}$ of cells in their maximal growth rate (same inoculum for all the bottles). The system was previously purged with $\mathrm{N}_{2}$. The temperature was fixed at $36{ }^{\circ} \mathrm{C}$ and gentle agitation was kept at $50 \mathrm{rpm}$. The whole system was autoclaved at $121{ }^{\circ} \mathrm{C}$ during 20 minutes before inoculation, $\mathrm{pH}$ value was set to 6 at the beginning of the fermentation and then it varied freely through the fermentation according to the acids generation and subsequent consumption.

Gas stripping fermentations were carried out by the application of four nitrogen stripping pulses of $1.5 \mathrm{vvm}$ for $30 \mathrm{~min}$ at $25,46,51$ and $118 \mathrm{~h}$ of fermentation, respectively. Schott bottles were adapted with a gas sparger in order to optimize the droplet distribution inside the bioreactor. A vegetable oil base mixture composed of sunflower oil (90\% v/v) and a $\mathrm{C}_{12}$ based Guerbet alcohol (2-Butyl -1-Octanol, 2B1O) (PubChem CID: 19800) was tested as extractive agent in biphasic fermentations. The ratio organic: aqueous phase was fixed at 1:1 (v/v). Low agitation allowed maintaining a clear separation between phases in all fermentations. The bioreactor was adapted with a sampling device for both aqueous and organic phases. The integrated system (GS+LLE) was carried out at the operating conditions described above. Two duplicates of each system were carried out at the same time. Only one of the duplicates was sampled periodically and the other one was kept closed and not sampled until the end of 204 fermentation. 
206 Samples were centrifuged at $4000 \mathrm{rpm}$ for $20 \mathrm{~min}$, therefore in the supernatant were 207 measured: pH (Toledo mettle; Columbus OH-USA), glucose consumption (YSI 2700 208 Select; Yellow Springs OH -USA). IBE products in the aqueous phase were quantified by Gas Chromatography (Agilent Technologies 7890B GC System; Santa Clara CA210 USA), equipped with an Agilent VF-624ms column using He (PubChem CID: 23987) as the carrier gas and a flame ionization detector (FID), temperature of the oven was 35 ${ }^{\circ} \mathrm{C}$ and it was increased at a gradient of $2{ }^{\circ} \mathrm{C} / \mathrm{min}$ until $60{ }^{\circ} \mathrm{C}$ and subsequently increased up $15{ }^{\circ} \mathrm{C} / \mathrm{min}$ to $200{ }^{\circ} \mathrm{C}$ for $10 \mathrm{~min}$. Alcohols were quantified in the organic phase with a back flush (reversal flow) system consisting of an $10 \mathrm{~m}$ HP-PONA precolumn (Agilent Technologies) with a pressure ramp of 38.1 psi for 30, 40, 45 and 50 min followed by $5 \mathrm{psi} / \mathrm{min}$ until $10 \mathrm{psi}$, and 0 psi during 0-10 min. The HP-PONA precolumn was connected to a 45 m HP-PONA column (Agilent Technologies) with a pressure of $34.5 \mathrm{psi}$ and a temperature ramp of $35{ }^{\circ} \mathrm{C}$ for $10 \mathrm{~min}, 1.1{ }^{\circ} \mathrm{C} / \mathrm{min}$ until 130 ${ }^{\circ} \mathrm{C}$, then $15{ }^{\circ} \mathrm{C} / \mathrm{min}$ until $280{ }^{\circ} \mathrm{C}$ and finally to $280{ }^{\circ} \mathrm{C}$ for $0-15 \mathrm{~min}$. The flame ionization detector temperature was at $300-310{ }^{\circ} \mathrm{C}$. Both columns used He as the carrier gas. In aqueous samples, free growing cell evolution was estimated measuring optical density at $600 \mathrm{~nm}$ (Spectrophotometer Shimadzu UV-1240; Kyoto, Japan).

\section{Results and Discussion}

\subsection{Butanol stripping rate}

226 The butanol stripping rate was investigated at three different gas flow rates: $0.25,1$ and $2271.5 \mathrm{~L} / \mathrm{min}$ (corresponding to $0.5,2$ and $3 \mathrm{vvm}$, respectively). The bioreactor was filled with synthetic aqueous solution representing the final IBE fermentation composition (11, 5 and $2 \mathrm{~g} / \mathrm{L}$ for butanol, isopropanol and ethanol, respectively) at the beginning of 
each experiment. Temperature was first raised up to $37^{\circ} \mathrm{C}$ inside the reactor and stirring rate was fixed at $300 \mathrm{rpm}$. Aqueous solution was sampled each $2 \mathrm{~h}$ for calculating alcohol striping rate. At the same time intervals, condensates were collected in the cold traps for further analyses and quantification. Experimental obtained data were first compared with SIMULIS thermodynamic modeling results for alcohols stripping rate inside the bioreactor (butanol aqueous concentration evolution in Fig. 2). It can be seen that thermodynamic prediction for aqueous butanol depletion rate is in good agreement with experimental data for 0.5 and $2 \mathrm{vvm}$. These results confirmed the absence of mass transfer limitation in the bioreactor under experimental conditions. In other words, thermodynamic controlled the process and therefore acquired data will give the maximal attainable butanol stripping rate or the minimal vvm that should be applied for a given extraction rate. Data obtained with 3 vvm showed that butanol was stripped slightly faster than thermodynamic prediction. This can be attributed to non-negligible physical entrainment of water droplets in outlet lines when high flowrates were applied.

Based on these experimental results and previous discussion, Eq. A.7 was regressed for estimation of stripping rate constant $\left(\beta, \mathrm{h}^{-1}\right)$ at different conditions (Table 1) as follows:

$$
\beta=\frac{\ln \left(\frac{\mathrm{C}_{\mathrm{i}, 0}}{\mathrm{Ci}}\right)}{\mathrm{t}}
$$

247 Butanol and isopropanol have similar stripping rate constants (slightly higher for butanol), and they are systematically two times higher than ethanol stripping rate constant. These results are in agreement with Vrije et al., 2013 [9], which studied the gas stripping with IBE model solutions at fixed $\mathrm{vvm}=1 \mathrm{~min}^{-1}$. Stripping rate from aqueous medium will depend not only on stripping coefficient (which encloses 
252 thermodynamics - Henry coefficient- and mass transfer -KLG-) but also on 253 concentration ratio of alcohols in aqueous system $[9,11]$. Stripping rate order has been 254 kept constant in our experiments: butanol was stripped higher than isopropanol, while 255 stripping rate of ethanol was lower. Relative stripping rate of alcohols is difficult to 256 predict as Henry coefficients from literature present high variability for these 257 compounds in such diluted solutions.

258 Alcohols stripping rates generally diminished with decreasing IBE concentrations in aqueous solution (Table 2). In batch process fermentations, butanol sets the inhibition threshold because it is the main inhibitory metabolite and its removal rate should be at 261 least equal or higher than the specific productivity of butanol in the bioreactor, in order 262 to avoid its accumulation. A macroscopic mode inspired in ABE literature was developed (data not shown) in order to estimate instantaneous butanol productivity in a batch fermentation. Maximal butanol productivity was close to $0.5 \mathrm{~g} / \mathrm{Lh}$ after $30 \mathrm{~h}$ of fermentation. These data were compared in Table 2 and it was observed for each gas flow rate applied (or vvm) a minimal butanol concentration in the aqueous phase inside the bioreactor that it was needed in order to equalize butanol productivity and butanol stripping rate. According to these results, a vvm=0.5 $\mathrm{min}^{-1}$ would never be enough to follow biological production in studied conditions $\left(37^{\circ} \mathrm{C}\right)$. Minimal concentration of 5 $\mathrm{g} / \mathrm{L}$ of butanol inside the bioreactor must be reached before applying a gas stripping of

$271 \mathrm{vvm}=2 \mathrm{~min}^{-1}$ in order to strip butanol as fast as its maximal productivity rate. Butanol 272 inhibition threshold imposes the maximal concentration that would be suggested in order to maximize productivity during operation. 


\subsection{Selectivity}

Selectivity is defined here as mass unity of stripped alcohol per mass unity of stripped water in the gas outlet of the bioreactor. In a batch operation system, selectivity varies as a function of water and inlet gas stripping composition, since the ratio water/alcohol in the gas stream is governed by system thermodynamics. In this experimental work, only end point selectivity is obtained, as a result of an overall mass balance of water and alcohol in the system; it would be therefore a mean value corresponding to the whole batch assay. In Fig. 3 selectivity was calculated by thermodynamic simulation (SIMULIS software, using specific in-house thermodynamic model) and was plotted as a function of butanol concentration in the aqueous phase inside the bioreactor at fixed operating conditions $\left(37^{\circ} \mathrm{C}\right.$ and $\left.2 \mathrm{vvm}\right)$. Experimental data corresponding to the end of the batch assay at $2 \mathrm{vvm}$ (at minimal butanol concentration) were higher than the estimated ones, since these data corresponded to the mean total selectivity considering higher concentration of butanol in the aqueous phase from the beginning of the experimental test, as it was stated previously.

Butanol selectivity by nitrogen stripping technique was low: which means that outlet gas left the bioreactor with non-negligible water quantity (even if physical entrainment was not considered here). This behavior will directly impact the operational cost of the process and will determine the recovery system of alcohols in gas loop.

Stripping rate of water scarcely varied in function of butanol aqueous concentration while stripping rate of butanol increased proportionally to its stripping coefficient and the local aqueous butanol composition [8]. Then, the asymptotic diminution of the process selectivity when the medium was increasingly diluted could be explained. These 
results showed that gas stripping process became interesting only with a fixed butanol concentration in the aqueous phase [11].

\subsection{Condensation rate and condensates composition}

301

302

303

In a gas stripping-fermentation coupled industrial process, not only stripping rate of the inhibitory metabolite should be kept at least equal to its production rate inside the bioreactor but also stripped alcohols should be fully recovered from the gas loop before being recycled, in order to renew and maintain their stripping capacity through the operation.

The experimental unit used in these experiments (Fig. 1) had two condensers in cascade working at 4 and $-15{ }^{\circ} \mathrm{C}$, respectively. Almost the totality of condensates were recovered from the first cold step at $4{ }^{\circ} \mathrm{C}$. Only at the end of each batch assay (when alcohol concentration was lower in the aqueous phase) a mass of condensates could be quantified from cold step at $-15{ }^{\circ} \mathrm{C}$. Strong linearity existed between butanol concentration in the condensates and butanol concentration in the aqueous phase (Fig. 4). Experimental data corresponding to GC analysis of condensates were obtained from the batch assays carried out at $v v m=2 \mathrm{~min}^{-1}$ (represented in Fig. 4) experimental data showed good agreement with the simulated ones obtained by SIMULIS model. Besides, if the aqueous butanol concentration was higher than its limit solubility $(7.7 \%$ wt. at 20 ${ }^{\circ} \mathrm{C}$ ) a phase demixing zone could force an additional separation of the condensate liquid phase (simulated data Fig. 4). Moreover, this behavior has been already proved in ABE fermentation-GS coupled technique [11]. Additionally, it would be possible to recover one or two liquid phase alcohols in the condensates collector (cold trap at $4{ }^{\circ} \mathrm{C}$ ) which were highly concentrated ( $>10-100$ times the initial concentration in the bioreactor) but the collected volume could represent only the $5 \%$ of bioreactor initial volume. 


\subsection{Suggested operation}

324 The first objective of the gas stripping recovery technique is the end product inhibition

325 alleviation by partial stripping of main inhibitory metabolites in a process. By means of 326 combined abiotic experimentation with synthetic fermentation broth and 327 thermodynamic simulation work, it has been proved that this technique becomes 328 interesting at higher alcohol concentration in aqueous phase (remained under inhibition 329 threshold). Indeed, not only butanol stripping rate was maximized when its 330 concentration was the highest in aqueous phase, but also selectivity of the process ( $\mathrm{g}$ 331 alcohol stripped/ g water stripped) decreased asymptotically when the medium was 332 diluted. In this work, alcohol concentration in condensates from the outlet gas was 333 linearly dependent on the stripped aqueous phase concentration and above solubility 334 limit of butanol in water $\left(\sim 7.7 \%\right.$ wt. at $\left.20^{\circ} \mathrm{C}\right)$ where additional separation by demixtion 335 zone appeared. From previous statements, it was suggested the application of gas 336 stripping technique for IBE fermentation in a pulse-mode (or intermittent mode): gas 337 stripping would be activated only when butanol attains a predefined concentration in 338 order to boost extraction performances.

3.5 Pulse gas stripping, extractive and integrated gas stripping-extractive coupling fermentations

342 IBE fermentations coupled to different separation techniques were carried out in batch conditions. GS, LLE and hybrid GS-LLE system are compared to control fermentation 344 (no separation technique). For GS and GS-LLE system, pulse-mode operation for gas 
injection (nitrogen) was applied at three predefined time intervals since aqueous butanol was not known on real time.

347 For extractive fermentations, a vegetable oil based mixture composed of sunflower oil 348 (90\% v/v) and a C12 based Guerbet alcohol (2 butyl-1octanol, 2B1O) was used as the extractive agent. Fig. 5 represents two discriminatory parameters in the performance of IBE fermentation with GS and LLE coupling techniques: the first one represents the glucose consumption and the second one the butanol concentration in aqueous phase. Synergic effect was reached with GS-LLE coupling technique based on higher glucose consumption rate and lower aqueous butanol concentration during the operation because butanol extraction rate from aqueous phase was more increased than individual ISPR techniques and control assays. Overconsumption of $23 \%$ glucose was observed when GS or LLE performed individually; while hybrid integrated technique showed $45 \%$ of overconsumption of glucose related to control fermentation. Total solvent concentrations were not quantified during these experiences because gas phase was not analyzed in these experiments. Nevertheless, total solvent IBE production could be estimated and ranked from sugar consumption and constant IBE yield of fermentation $(0.35 \mathrm{~g} / \mathrm{g})$. These data are synthetized in Table 3. On the other hand, in Fig. 5 it is observed that bioactivity was stopped approximately at $60 \mathrm{~h}$ for fermentations containing the extracting phase inside the bioreactor, even if non-inhibitory butanol concentration $(<4 \mathrm{~g} / \mathrm{L})$ was measured in the aqueous phase and remaining glucose concentration could be quantified. This could be attributed to midterm toxicity of the solvent used in LLE-fermentations towards the specific microorganism employed. This needs to be further investigated. This solvent showed biocompatibility at the beginning of the fermentation in previous screening work (data not shown). Glucose consumption 
rate and biomass formation were enhanced during the first $50 \mathrm{~h}$ (Fig. 5), which means that biocompatibility of this solvent is not an issue during the first part of the

371 fermentation. Kollerup and Daugulis (1985) [15] classified the modes of cell inhibition

372 in extractive fermentations into different mechanisms depending on the initial effect of

373 the solvent into the metabolic and enzymes activity during the fermentation. In our case, middle term toxicity was observed with vegetable oil mixture and $2 \mathrm{~B} 1 \mathrm{O}$, respectively. These results should be confirmed in future experiments.

Respective theoretical equilibrium concentrations of butanol and isopropanol in organic and aqueous phase are estimated from experimental partition coefficient of the extracting solvent previously determined and considered organic and aqueous volumes at each sampling time. These data were plotted in Fig. 6 with experimental data corresponding to the evolution and distribution of butanol and isopropanol concentrations in aqueous and organic phases inside the biphasic bioreactor. Both series data (experimental -lines- and theoretical equilibrium distribution -dots-) were in agreement so it could be concluded that GS-LLE system behaved as an equilibrium stage in the experimental conditions studied for this particular set up configuration. In other words, the mass liquid-liquid transfer rate was higher than the main metabolite production rate. This behavior has already been observed for ABE fermentation [12]. On the other hand, butanol in organic phase in hybrid GS-LLE system seemed to be partially stripped when compared to LLE single technique, since its concentration slightly decreased from $50 \mathrm{~h}$ of fermentation. This can be explained by a phase transfer phenomena from organic phase to aqueous phase while aqueous phase has been stripped. Butanol in aqueous phase forms butanol/water azeotrope which is more volatile than butanol and water alone. When gas stripping technique was applied, the 
azeotrope was stripped because of its lower boiling point than water. Interestingly,

394

395

396

397

398

399

400

401

402

403

404

405

406

407

408

409

410

411

412

413

414

415

416

organic phase acted in this case as a butanol storage to both limit and control butanol in aqueous phase, while gas stripping technique removed the extra water concentration. The concomitant gas stripping with this configuration has already been mentioned [14]. The authors applied in their system oleyl alcohol as an extracting agent, which has four times higher partition coefficient for butanol than the vegetable oil based mixture used in this work. As a result, it had a positive effect for liquid extraction but made it more difficult for solvent regeneration (more alcohol affinity in organic phase). Continuous gas stripping technique was applied directly in the organic phase system from $48 \mathrm{~h}$ of fermentation in order to boost final glucose consumption, while pulse-fedbatch gas stripping was applied in this work from the early step of fermentation in order to increase glucose consumption and production rates.

\section{Conclusions}

Gas stripping was studied in abiotic representative system for (A)IBE batch fermentation. High flow rates $\left(\mathrm{vvm}>2 \mathrm{~min}^{-1}\right)$ were needed in order to achieve stripping rate of butanol higher than biological production rate. A pulse-GS mode was suggested, allowing to maximize selectivity (g butanol/g water stripped) and alcohol concentration in condensates. Combined separation techniques (pulse -GS-LLE) were then applied to batch fermentations. A synergic effect appeared when using the integrated technique, resulting in the highest butanol extraction rate and productivity. Moreover, biphasic bioreactor acted as an equilibrium step and both phases were stripped concomitantly. 
418 [1] Jones DT, Woods DR, Acetone-Butanol Fermentation Revisited Microbiol Rew $419 \quad 1986 ; 50(4): 484-524$.

420

[2] Oudshoorn A, Van der Wielen LAM, Straathof AJJ. Assessment of Options for 422 Selective 1-Butanol Recovery from Aqueous Solution. Ind Eng Chem Res 2009;48:7325-7336.

424

[3] Leland MV. Separation technologies for the recovery and dehydration of alcohols from fermentation broths. Biofuels Bioprod Bioref 2008;2(6):553-588.

[4] Woodley JM, Bisschops M, Straathof AJJ. Ottens M. Perspective Future directions for in-situ product removal (ISPR). J Chem Technol Biotechnol 2008;83:121-123.

430

[5] Qureshi N, Hughes S, Maddox IS, Cotta MA. Energy-efficient recovery of butanol 432 from model solutions and fermentation broth by adsorption. Bioprocess Biosyst Eng $433 \quad 2005 ; 27: 215-222$.

[6] Groot WJ, Van der Lans RGJM, Luyben KCAM. Technologies for Butanol

[7] Qureshi N, Blaschek HP. Recovery of butanol from fermentation broth by gas stripping. Renew. Energy. 2001;22:557-564.

[8] Liao YC, Lu KM, Li SY. Process parameters for operating 1-butanol gas stripping in a fermentor. Journal Bioscience Bioeng 2014;1-7.

[9] De Vrije T, Budde M, Van der Wal H, Claassen PAM, López-Contreras AM. In situ 445 removal of isopropanol, butanol and ethanol from fermentation broth by gas stripping. 446 Bioresource Technol 2013;137:153-159. 
448 [10] Ezeji TC, Karcher PM, Qureshi N, Blaschek HP. Improving performance of a gas 449 stripping-based recovery system to remove butanol from Clostridium beijerinckii 450 fermentation. Bioprocess Biosyst Eng 2005;27:207-214.

451

452 [11] Xue C, Zhao J, Lu C, Yang ST, Bai F, Tang IC. High-titer n-butanol production by 453 Clostridium acetobutylicum JB200 in fed-batch fermentation with intermittent gas 454 stripping. Biotechnol Bioeng 2012;109(11):2746- 2756.

455

456 [12] González-Peñas H, Lu-Chau TA, Moreira MT, Lema JM. Solvent screening 457 methodology for in situ ABE extractive fermentation. Appl Microbiol Biotechnol 2014; 458 98 (13):5915-5924.

459

460 [13] Lu KM, Li SY. An integrated in situ extraction-gas stripping process for Acetone461 Butanol-Ethanol (ABE) fermentation. J. Taiwan Inst Chem Eng 2014;45:2106-2110.

462

463

[14] Lu KM, Chiang YS, Wang YR, Chein RY, Li SY. Performance of fed-batch 464 acetone-butanol-ethanol (ABE) fermentation coupled with the integrated in situ 465 extraction gas stripping process and the fractional condensation. J Taiwan Inst Chem 466 Eng 2016;60:119-123.

467

468

469

[15] Kollerup F, Daugulis AJ. Screening and identification of extractive fermentation 470

471

472

473 
482 Table 1. Stripping rate constant $(\beta)$ at $0.5,2$ and $3 \mathrm{vvm}$ for synthetic IBE aqueous 483 solutions.

484

485 Table 2. Butanol removal rate from model.

486

487 Table 3. Estimated IBE concentration $(\mathrm{g} / \mathrm{L})$ in aqueous phase under ISPR (In situ 488 product recovery technique): gas stripping (GS), liquid-liquid extraction (LLE) and 489 coupling GS-LLE fermentations.

490 
491 Fig.1. Experimental system for abiotic IBE gas stripping used in this study.

492

493 Fig.2. Butanol aqueous concentration evolution (experimental data: dots, 494 thermodynamic simulation: lines) $\mathrm{vvm}=0.5 \min ^{-1}(\boldsymbol{\square}) ; \mathrm{vvm}=2 \min ^{-1}(\mathbf{\Lambda}) ; \mathrm{vvm}=3 \mathrm{~min}^{-1}$ 495

496

497 Fig.3. Selectivity variation of the batch abiotic gas stripping process, experimental data 498

(匹), thermodynamic simulation (line).

499

500 Fig.4. Condensates composition at $4{ }^{\circ} \mathrm{C}$ versus aqueous butanol concentration, 501 experimental data $(\boldsymbol{\Delta})$, total condensate simulation (line), demixed condensates 502 simulation (o).

503

504

Fig.5. Glucose, butanol concentration and absorbance in aqueous phase with ISPR

505 techniques. ( $\Delta \mathrm{GS}, \diamond \mathrm{LLE}, \bullet \mathrm{GS}+\mathrm{LLE}, \mathrm{x}$ control).

506

507 Fig.6. Total butanol and isopropanol (g/L) concentration in aqueous and organic phases 508 with LLE and GS-LLE coupling fermentations. Calculated butanol (०); calculated 509 isopropanol $(\Delta)$; experimental butanol $(\bullet)$; experimental isopropanol $(\boldsymbol{\Delta})$.

510

511

512 
515 Table 1. Stripping rate constant $(\beta)$ at $0.5,2$ and 3 vvm for synthetic IBE aqueous 516 solutions.

\begin{tabular}{|c|c|c|c|}
\hline \multirow[b]{2}{*}{ Alcohols } & \multicolumn{3}{|c|}{ Stripping rate constant, $\beta\left(h^{-1}\right)$} \\
\hline & vvm $0.5 \mathrm{~min}^{-1}$ & vvm $2 \min ^{-1}$ & vvm 3 min $^{-1}$ \\
\hline Butanol & $0.042 \pm 0.009$ & $0.107 \pm 0.009$ & $0.184 \pm 0.010$ \\
\hline Isopropanol & $0.040 \pm 0.01$ & $0.098 \pm 0.009$ & $0.170 \pm 0.002$ \\
\hline Ethanol & $0.021 \pm 0.005$ & $0.053 \pm 0.006$ & $0.108 \pm 0.010$ \\
\hline
\end{tabular}

517

518

519 Table 2. Butanol removal rate from model.

\begin{tabular}{|c|c|c|c|}
\hline \multirow{2}{*}{$\begin{array}{l}\text { Butanol concentration in aqueuse phase } \\
\qquad(\mathrm{g} / \mathrm{L})\end{array}$} & \multicolumn{3}{|c|}{ Butanol stripping rate (g/Lh) } \\
\hline & $v v m=0,5 \mathrm{~min}^{-1}$ & $\mathrm{vvm}=2 \mathrm{~min}^{-1}$ & $\mathrm{vvm}=3 \mathrm{~min}^{-1}$ \\
\hline 0 & 0 & 0 & 0 \\
\hline 2 & 0.08 & 0.21 & 0.37 \\
\hline 4 & 0.17 & 0.43 & 0.74 \\
\hline 6 & 0.25 & 0.64 & 1.10 \\
\hline 8 & 0.34 & 0.86 & 1.47 \\
\hline 10 & 0.42 & 1.07 & 1.84 \\
\hline
\end{tabular}

520

521 Table 3. Estimated IBE concentration $(\mathrm{g} / \mathrm{L})$ in aqueous phase under ISPR (In Situ 522 Product Recovery technique): gas stripping (GS), liquid-liquid extraction (LLE) and 523 coupling GS-LLE fermentations.

524

\begin{tabular}{|l|c|}
\hline & $\begin{array}{c}\text { Estimated IBE concentration } \\
(\mathbf{g} / \mathbf{L})\end{array}$ \\
\hline Control & $12.41 \pm 0.38$ \\
\hline GS & $13.01 \pm 1.61$ \\
\hline LLE & $13.82 \pm 0.65$ \\
\hline GS+LLE & $16.46 \pm 0.24$ \\
\hline
\end{tabular}




\section{$526 \quad$ Figures}

527

528

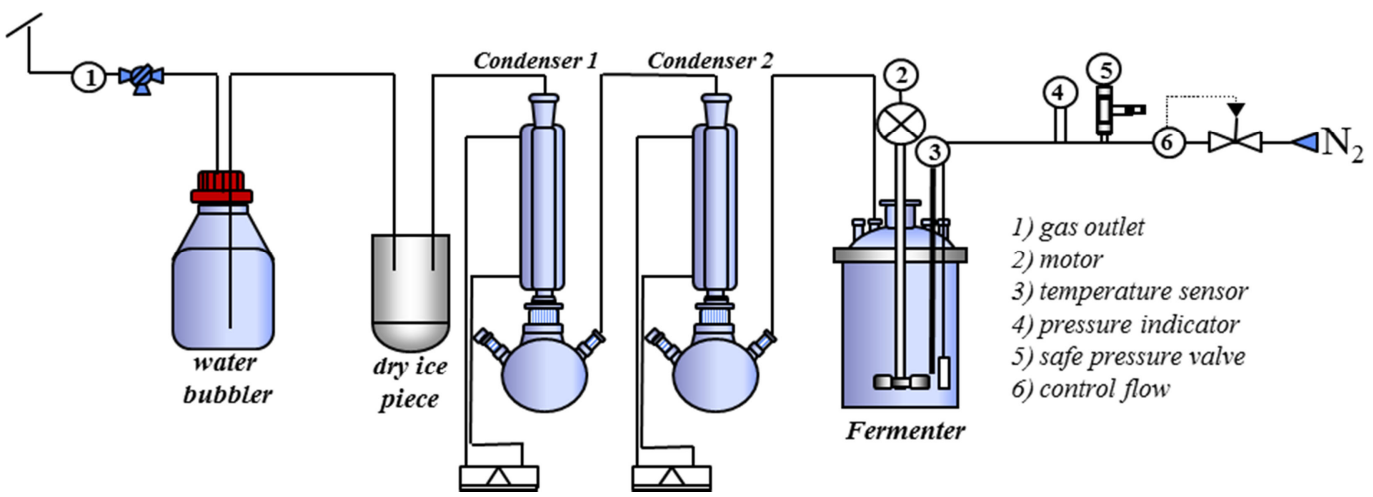

529 Fig. 1. Experimental system for abiotic IBE gas stripping used in this study.

530

531 


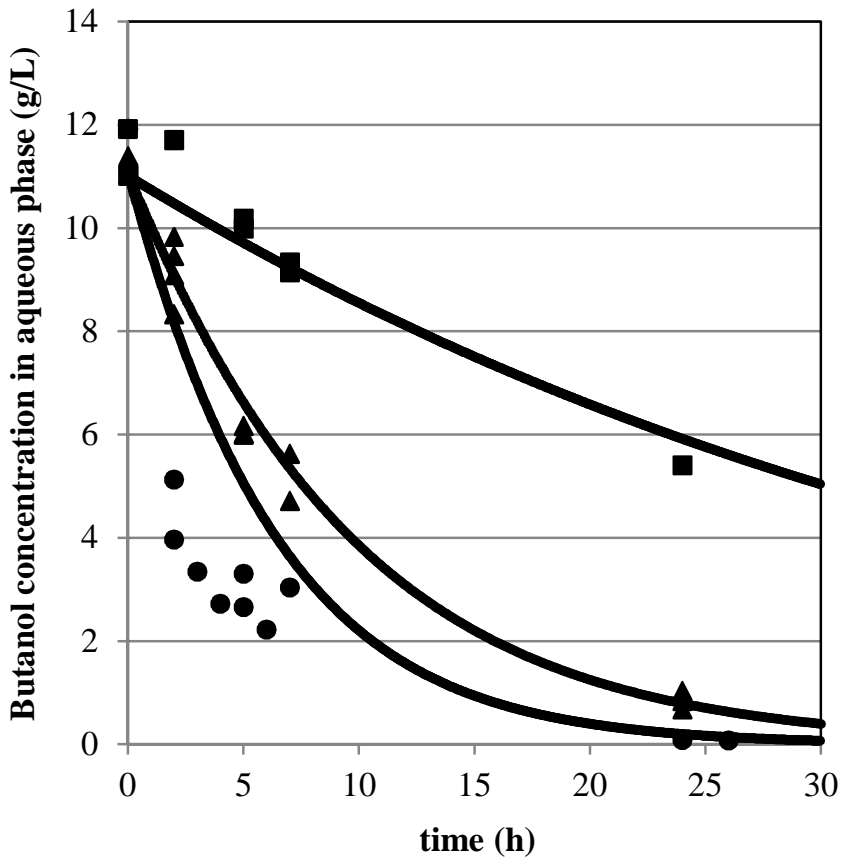

532

533 Fig. 2. Butanol aqueous concentration evolution (experimental data: dots,

534 thermodynamic simulation: lines) $\mathrm{vvm}=0.5 \min ^{-1}(\boldsymbol{\square}) ; \mathrm{vvm}=2 \min ^{-1}(\boldsymbol{\Delta})$; vvm=3 $\mathrm{min}^{-1}$ 535 $(\bullet)$.

536 


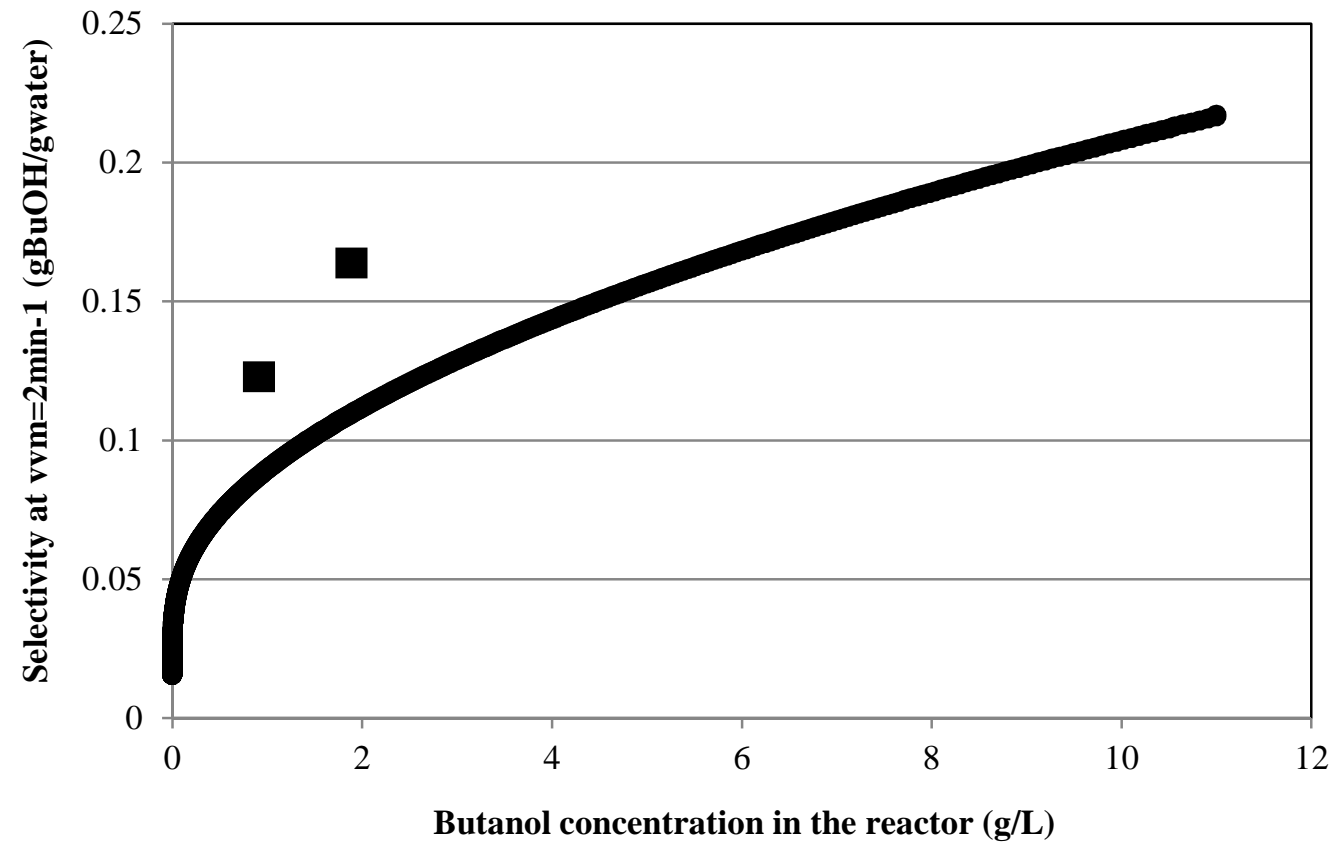

537

538 Fig. 3. Selectivity variation of the batch abiotic gas stripping process, experimental data

539 (匹), thermodynamic simulation (line). 


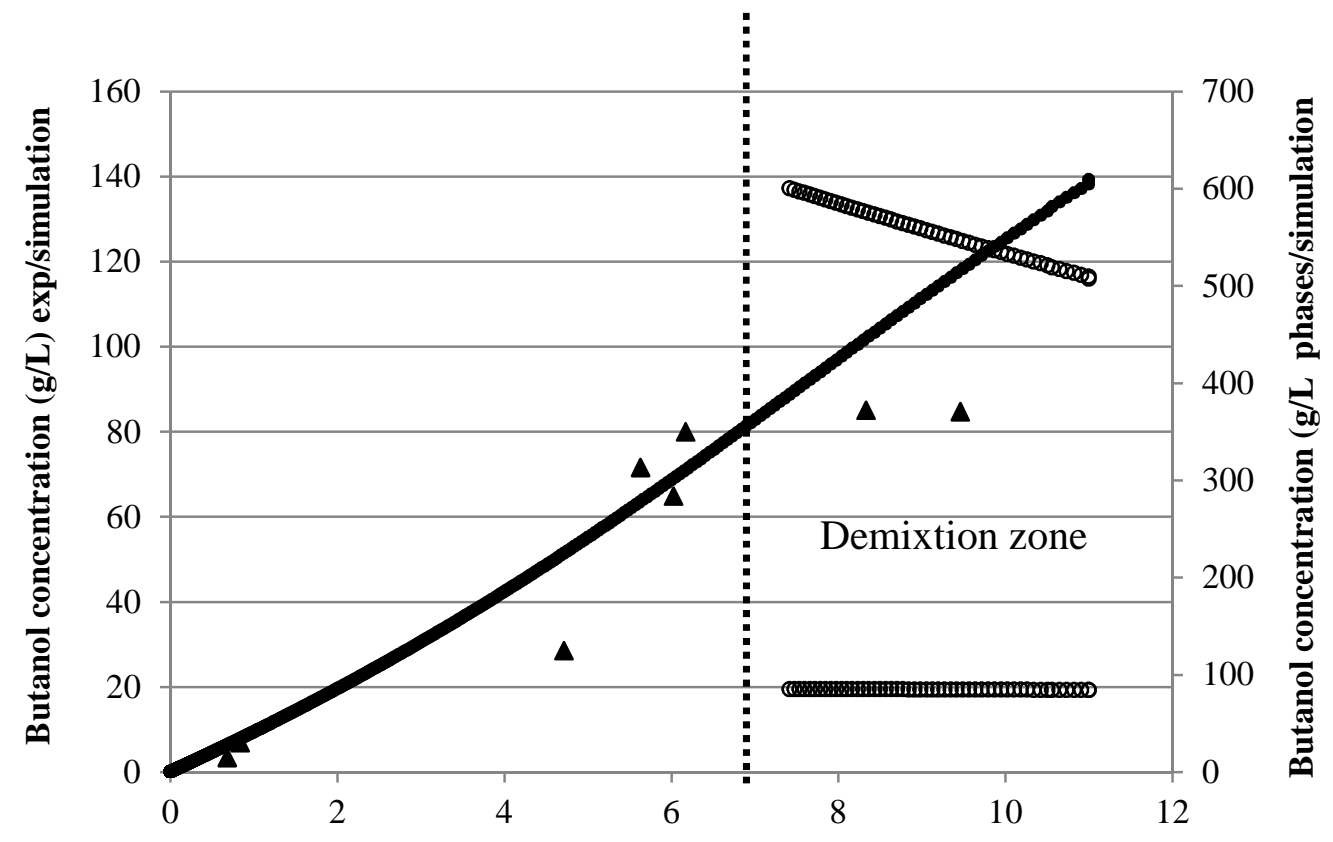

542

Butanol concentration in the reactor $(\mathrm{g} / \mathrm{L})$

543 Fig. 4. Condensates composition at $4{ }^{\circ} \mathrm{C}$ versus aqueous butanol concentration,

544 experimental data $(\boldsymbol{\Delta})$, total condensate simulation (line), demixed condensates 545 simulation (o). 

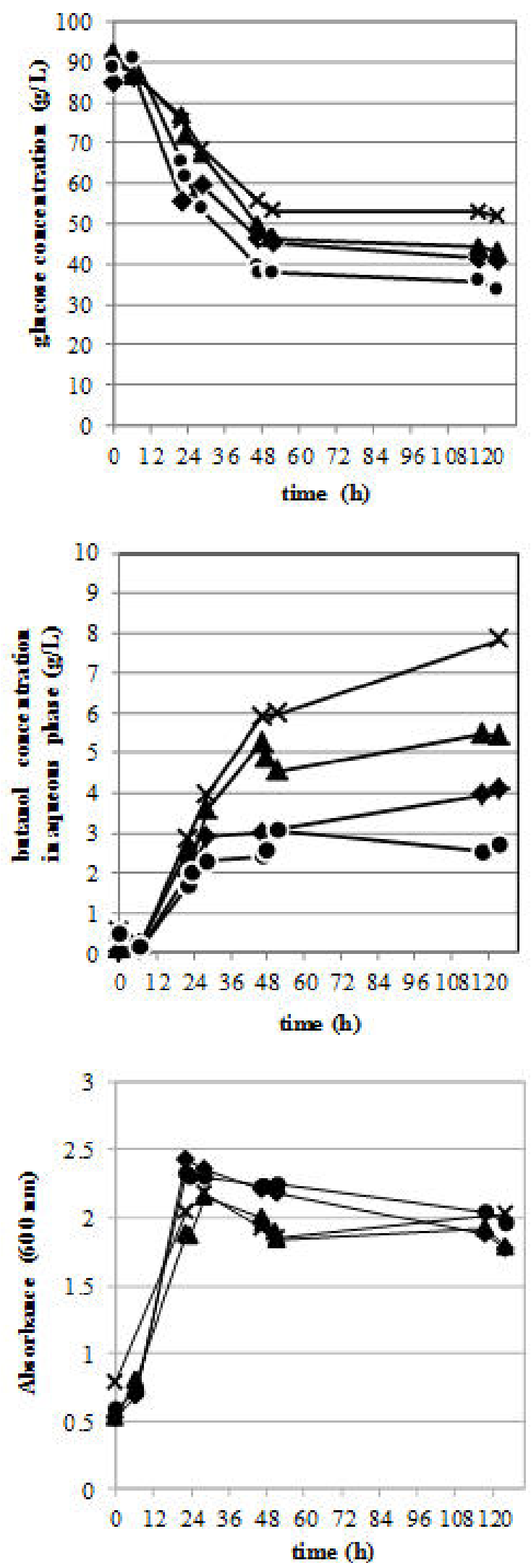

556 Fig. 5. Glucose, butanol concentration and absorbance in aqueous phase with ISPR 557 techniques. ( $\Delta$ GS, $\bullet$ LLE, $\bullet$ GS-LLE, $x$ control). 



560 Fig. 6. Total butanol and isopropanol $(\mathrm{g} / \mathrm{L})$ concentration in aqueous and organic phases

561 with LLE and GS-LLE coupling fermentations. Calculated butanol (०); calculated 562 isopropanol $(\Delta)$; experimental butanol $(\bullet)$; experimental isopropanol $(\boldsymbol{\Delta})$. 


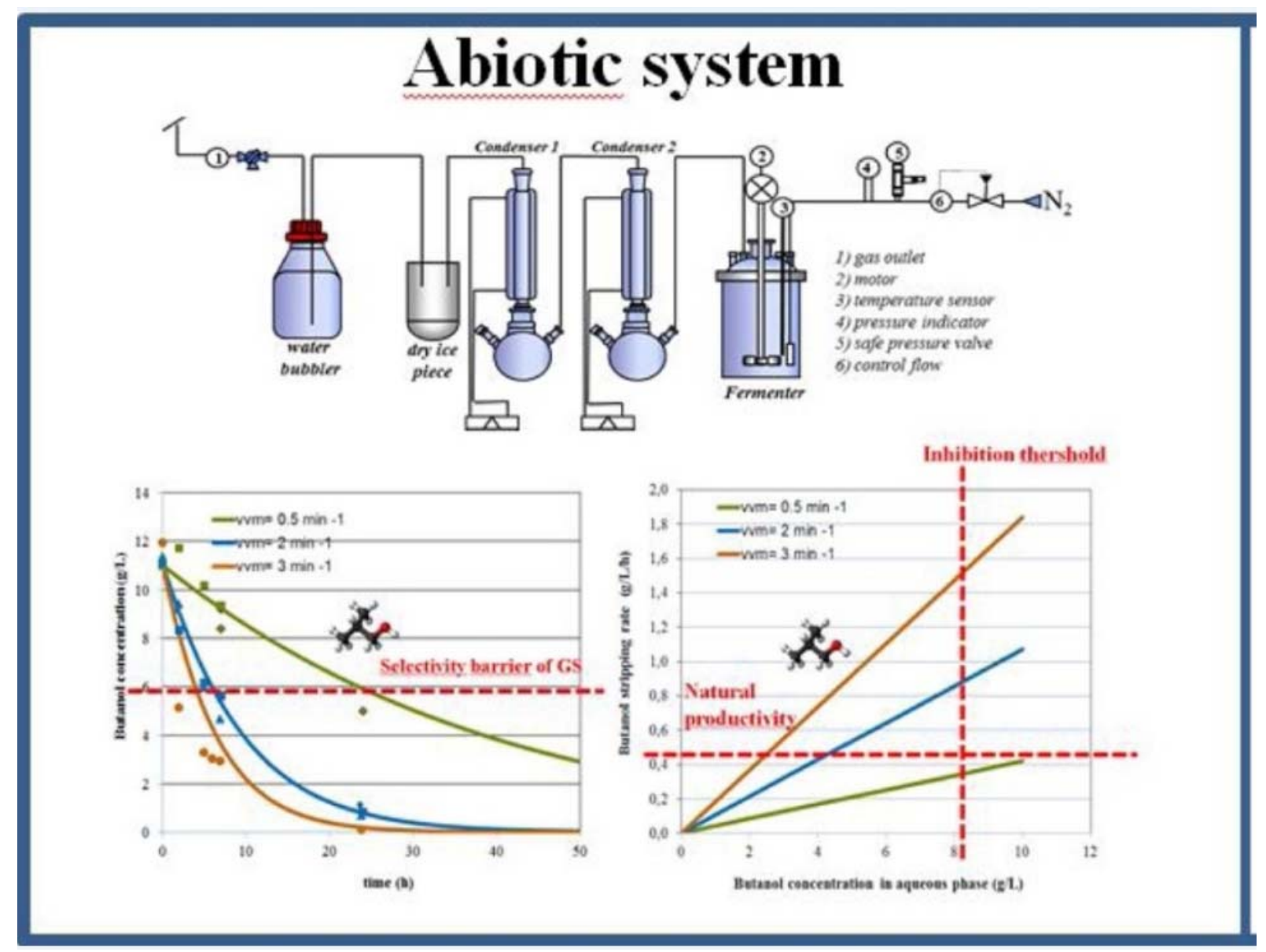




\section{Integrated GS+LLE}
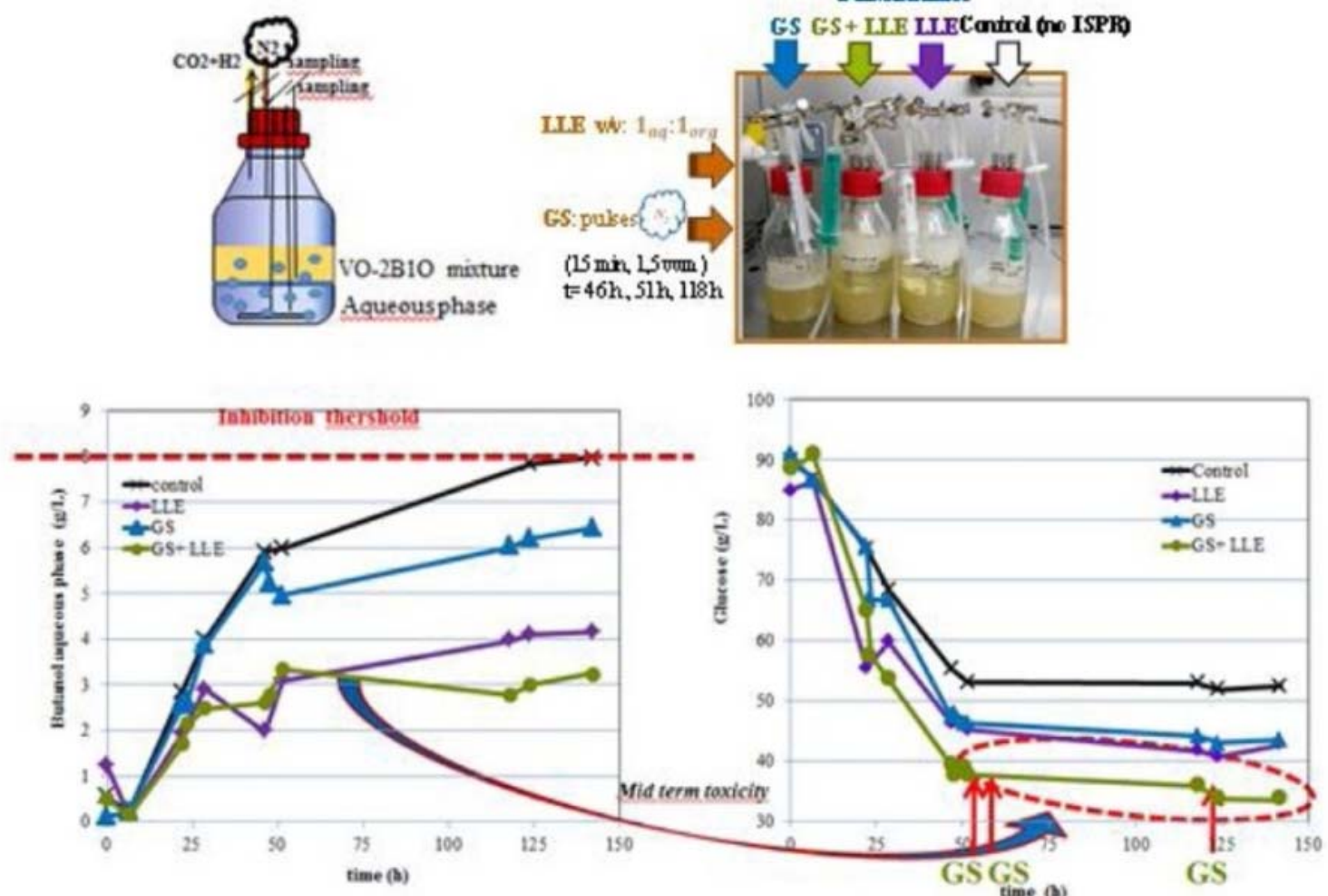